\title{
Coronavirus Disease 2019 Infection in a Child with Mild Symptoms and Atypical Course
}

\author{
Elif Koç ${ }^{1}$ Şule Yıldırım² \\ ${ }^{1}$ Department of Pediatrics, Burdur Bucak State Hospital, Burdur, Turkey \\ 2 Department of Pediatrics, Uşak Medical Park Hospital, Uşak, Turkey \\ J Pediatr Infect Dis 2021;16:89-90.
}

\begin{abstract}
Address for correspondence Sule Yıldırım, MD, Department of Pediatrics, Uşak Medical Park Hospital, Uşak 64100, Türkiye (e-mail: sulesIn@yahoo.com).
\end{abstract}

\begin{abstract}
Keywords

- coronavirus

- maculopapular rash

- pandemic

The number of children infected with new type coronavirus disease 2019 (COVID-19) is relatively small; its atypical, mild, and unexpected clinical pictures can be suggested as the reason. In this article, we reported a 25-month-old girl patient without any symptom besides high fever and who had no risk factor or suspicious contact. Also, she developed maculopapular rash that is not reported before in the COVID-19 patients to the best of our knowledge. In the pandemic states, any symptom should be evaluated as potential COVID-19 and detection and isolation of these cases are necessary as it can affect dissemination.
\end{abstract}

\section{Introduction}

Coronavirus disease (COVID-19) was first reported at the end of 2019, rapidly spread all around the world in a few months, and was declared a worldwide pandemic by World Health Organization in March 2020. As a novel infectious disease, it affects respiratory tract primarily and in some conditions may result in acute respiratory distress syndrome, multiorgan failure, and even death. ${ }^{1}$ It is known that it affects children relatively less than adults and the rate of the sick children is $2 \%{ }^{2}$ It is not clear yet whether this is due to the lower risk in children or to the fact that they had fewer symptoms. Although the most important factors affecting the mortality and morbidity are age and comorbidities, we may encounter conditions in children requiring aggressive treatment such aseptic shock, metabolic acidosis, irreversible bleeding, and coagulation dysfunction. ${ }^{3}$ In fact, atypical symptomatology may cause misdiagnosis or under diagnosis. Herein, we presented a case with fever and no more symptoms or risk factors who in the follow-up developed maculopapular rash that, to the best of our knowledge, is not reported before in the COVID-19 patients.

\section{Case Report}

A 25-month-old girl presented to outpatient clinic with high fever. She had fever for 3 days and it was approximately $39^{\circ} \mathrm{C}$.

received

May 9, 2020

accepted after revision

October 28, 2020

published online

December 7, 2020

There were no other symptoms as rhinitis or cough. She vomited once 3 days before. There was no risky contact; she and her family were isolated at home. Her 12-year-old sister had fever 1 week before but recovered without any treatment. In the past medical history, she was a term baby with normal motor mental development, her vaccination schedule was compatible with her age and there was no known chronic diseases. On the physical examination, there was not any sign other than oropharyngeal hyperemia. The laboratory tests showed leukopenia $(3,180 / \mu \mathrm{L})$, lymphopenia $(890 /$ $\mu \mathrm{L})$, thrombocytopenia $(91,000 / \mu \mathrm{L})$, and elevated liver function tests (AST 110, ALT 115, GGT 93, and LDH $339 \mathrm{U} / \mathrm{L}$ ). Serum electrolytes were as $\mathrm{Na} 130 \mathrm{mmol} / \mathrm{L}, \mathrm{K} 3,7 \mathrm{mmol} / \mathrm{L}$, and $\mathrm{Cl} 94,9 \mathrm{mmol} / \mathrm{L}$. Procalcitonin, CRP, and D-dimer values were high (4 ng/mL [reference value 0-0.5], 11,467 mg/L [05], and $1,179 \mathrm{ng} / \mathrm{mL}$ [0-500], respectively). Prothrombin time was 18.2 seconds and international normalized ratio was 1.67. Urine examination and chest X-ray were normal. Blood and urine culture were negative for bacterial infections. Pharyngeal swab was taken for new type coronavirus infection and cefotaxime, azithromycin, and oseltamivir were given as a treatment. In the following day, she developed maculopapular rashes all over the body ( - Fig. 1 ). The rash was unresponsive to antihistaminic and dexamethasone treatments and increased over time. Viral parameters for hepatitis B, CMV, EBV, rubella, and measles were studied and

(c) 2020. Thieme. All rights reserved. Georg Thieme Verlag KG, Rüdigerstraße 14, 70469 Stuttgart, Germany
DOI https://doi.org/ 10.1055/s-0040-1721447. ISSN $1305-7707$. 


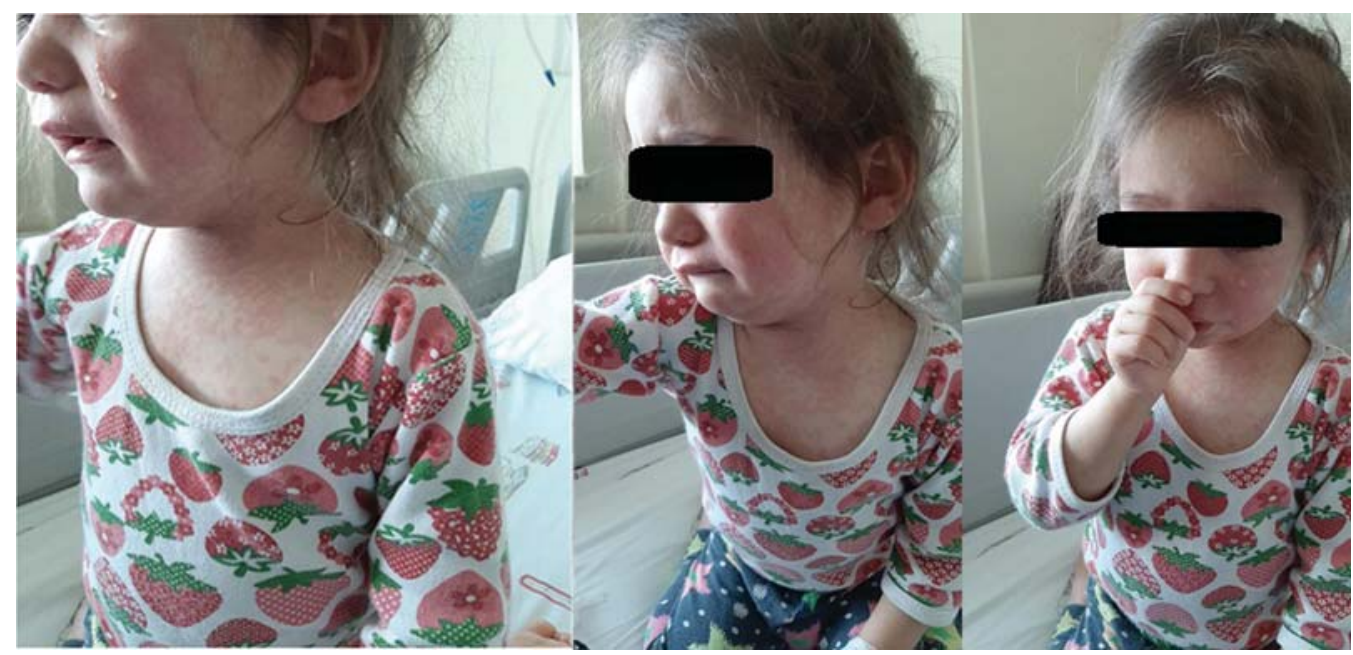

Fig. 1 The maculopapular rash of the patient.

resulted negative. The pharyngeal swab was positive for COVID-19. The other family members were tested negative after that result, but the family was isolated after the patient hospitalized. Hydroxychloroquine was added to treatment. Her abdominal ultrasonography and thorax CT were normal. In the following laboratory tests, D-dimer was 3,600 ng/mL, procalcitonin was $90 \mathrm{ng} / \mathrm{mL}$, and CRP was $60 \mathrm{mg} / \mathrm{L}$; there still was lymphopenia $(500 / \mu \mathrm{L})$ and thrombocytopenia $(90,000 / \mu \mathrm{L})$. She was referred to the pediatric infectious diseases clinic. At the follow-up, the laboratory parameters began to normalize and in the 7th day repeated pharyngeal swab was negative, laboratory parameters were normal, and the rash disappeared. She was not given IVIG or lopinavir/ ritonavir. After recovery, she was discharged. She and her family were isolated for 14 days.

\section{Discussion}

Although our patient presented only with high fever and there was no any risk factor or risky contact, we suspected from COVID-19, tested our patient, and found positive results considering that there may be an atypical course in children. This has supported the idea that an atypical symptomatology may cause misdiagnosis or under diagnosis in children.

In the COVID-19 infected children, the most common symptoms are fever, cough, fatigue, nasal congestion, rhinorrhea, sputum, and diarrhea. ${ }^{3}$ The fever is low grade in most of the cases. In our patient, the fever was high grade and resistant. She presented one episode of vomiting, but the other typical symptoms were not seen. In the 2nd day of follow-up, the patient developed maculopapular rash that is reported seldom before in the COVID-19 children to the best of our knowledge in the time and reported our case. Morey-Olivé et al reported two similar cases with infection by SARS-CoV-2 with cutaneous manifestations. ${ }^{4}$ Also, in time manner after our case, in some children infection manifesting as a hyper inflammatory syndrome with multiorgan involvement similar to Kawasaki disease shock syndrome was noted. ${ }^{5}$ Therefore, in the pandemic states, it will be logic to consider rash as a sign of COVID-19 until proven otherwise.

Isolation of the COVID-19 patients is substantial to slow the rate of transmission of the disease. It should be remembered that children can spread the disease like adults. In our case, the other family members were tested negative, but the old sister had fever a week before therefore may become negative at that time our patient tested. For this reason, keeping the index of suspicion high when approaching pediatric patients should not be ignored within the measures to be taken to prevent the spread of this disease. Even if there is no risk factor and case contact, there may be an atypical course in children and the findings should be evaluated well.

\section{Funding}

None.

Conflict of Interest

None declared.

\section{References}

1 Tezer H, Bedir Demirdağ T. Novel coronavirus disease (COVID-19) in children. Turk J Med Sci 2020;50(SI-1):592-603

$2 \mathrm{Wu} \mathrm{Z}$, McGoogan JM. Characteristics of and important lessons from the coronavirus disease 2019 (COVID-19) outbreak in China: summary of a report of 72314 cases from the Chinese center for disease control and prevention. JAMA 2020

3 Choi SH, Kim HW, Kang JM, Kim DH, Cho EY. Epidemiology and clinical features of coronavirus disease 2019 in Children. ClinExpPediatr 2020

4 Morey-Olivé M, Espiau M, Mercadal-Hally M, Lera-Carballo E, García-Patos V. Cutaneous manifestations in the current pandemic of coronavirus infection disease (COVID 2019). An Pediatr (Engl Ed) 2020;92(06):374-375

5 Riphagen S, Gomez X, Gonzalez-Martinez C, Wilkinson N, Theocharis P. Hyperinflammatory shock in children during COVID-19 pandemic. Lancet 2020;395(10237):1607-1608 